\title{
Cytoplasmic localization of Hug1p, a negative regulator of the MEC1 pathway, coincides with the compartmentalization of Rnr2p-Rnr4p
}

William B. Ainsworth

Michael G. Benton

Bridget Todd Hughes

Oliver Kerscher

William \& Mary

\section{Recommended Citation}

Ainsworth, W. B., Hughes, B. T., Au, W. C., Sakelaris, S., Kerscher, O., Benton, M. G., \& Basrai, M. A. (2013). Cytoplasmic localization of Hug1p, a negative regulator of the MEC1 pathway, coincides with the compartmentalization of Rnr2p-Rnr4p. Biochemical and biophysical research communications, 439(4), 443-448.

This Article is brought to you for free and open access by the Arts and Sciences at W\&M ScholarWorks. It has been accepted for inclusion in Arts \& Sciences Articles by an authorized administrator of W\&M ScholarWorks. For more information, please contact scholarworks@wm.edu. 


\title{
Cytoplasmic localization of Hug1p, a negative regulator of the MEC1 pathway, coincides with the compartmentalization of Rnr2p-Rnr4p
}

\author{
William B. Ainsworth ${ }^{\mathrm{a}, 1}$, Bridget Todd Hughes ${ }^{\mathrm{b}, 1}$, Wei Chun Au ${ }^{\mathrm{b}}$, Sally Sakelaris ${ }^{\mathrm{b}}$, Oliver Kerscher ${ }^{\mathrm{c}}$, \\ Michael G. Benton ${ }^{\mathrm{a}, *}$, Munira A. Basrai ${ }^{\mathrm{b}, *}$ \\ ${ }^{a}$ Cain Department of Chemical Engineering, Louisiana State University, Baton Rouge, LA 70803, USA \\ ${ }^{\mathrm{b}}$ Genetics Branch, Center for Cancer Research, National Cancer Institute, National Institutes of Health, Bethesda, MD 20892, USA \\ ${ }^{\mathrm{c} B i o l o g y}$ Department, The College of William E' Mary, Williamsburg, VA 23185, USA
}

\section{A R T I C L E I N F O}

\section{Article history:}

Received 16 August 2013

Available online 5 September 2013

\section{Keywords:}

Saccharomyces cerevisiae

HUG1

MEC1

$R N R$

HU arrest

DNA damage

Subcellular localization

\begin{abstract}
A B S T R A C T
The evolutionarily conserved MEC1 checkpoint pathway mediates cell cycle arrest and induction of genes including the RNR (ibonucleotide reductase) genes and HUG1 (ydroxyurea, ultraviolet, and gamma radiation) in response to DNA damage and replication arrest. Rnr complex activity is in part controlled by cytoplasmic localization of the Rnr2p-Rnr4p subunits and inactivation of negative regulators Sml1p and Dif1p upon DNA damage and hydroxyurea (HU) treatment. We previously showed that a deletion of $H U G 1$ rescues lethality of mec1 $\Delta$ and suppresses dun $1 \Delta$ strains. In this study, multiple approaches demonstrate the regulatory response of Hug1p to DNA damage and HU treatment and support its role as a negative effector of the MEC1 pathway. Consistent with our hypothesis, wild-type cells are sensitive to DNA damage and HU when HUG1 is overexpressed. A Hug1 polyclonal antiserum reveals that HUG1 encodes a protein in budding yeast and its MEC1-dependent expression is delayed compared to the rapid induction of Rnr3p in response to HU treatment. Cell biology and subcellular fractionation experiments show localization of Hug1p-GFP to the cytoplasm upon HU treatment. The cytoplasmic localization of Hug1p-GFP is dependent on MEC1 pathway genes and coincides with the cytoplasmic localization of Rnr2p-Rnr4p. Taken together, the genetic interactions, gene expression, and localization studies support a novel role for Hug $1 \mathrm{p}$ as a negative regulator of the $M E C 1$ checkpoint response through its compartmentalization with Rnr2p-Rnr4p.
\end{abstract}

Published by Elsevier Inc.

\section{Introduction}

Cellular survival in response to DNA lesions and replication arrest requires the coordination of checkpoint-mediated mechanisms to ensure DNA damage repair, cell cycle arrest, and recovery for genome stability. Checkpoint pathways regulate the expression of protein kinases, which mediate a transcriptional response and cell cycle arrest through downstream effectors. In Saccharomyces cerevisiae, the evolutionarily conserved MEC1 (ortholog to the

Abbreviations: BLM, bleomycin; DEX, dextrose; dNTP, deoxyribonucleotide; GAL, galactose; GFP, green fluorescent protein; HU, hydroxyurea; MMS, methy methanesulfonate; PBS, phosphate buffered saline; Rnr, ribonucleotide reductase.

* Corresponding authors. Address: Cain Department of Chemical Engineering, Louisiana State University, 110 Chemical Engineering, S. Stadium Rd., Baton Rouge, LA 70803, USA (M.G. Benton). Address: Genetics Branch, Center for Cancer Research, National Institutes of Health, 41 Medlars Drive, Bethesda, MD 20892, USA. Fax: +1 3014800380 (M.A. Basrai).

E-mail addresses: benton@lsu.edu (M.G. Benton), basraim@mail.nih.gov (M.A. Basrai).

1 These authors contributed equally to this work. human ataxia telangiectasia mutated- and Rad3-related - ATR protein) checkpoint pathway regulates origin firing, fork progression, and DNA repair and recovery (reviewed in [1]).

Mec1p and its effector kinases, Rad53p and Dun1p, activate both positive and negative effectors that regulate deoxyribonucleotide (dNTP) pools, cell cycle arrest, and recovery [1]. The activity of the positive effector RNRs (Ribonucleotide reductases), which are responsible for the rate-limiting conversion step of ribonucleotides (rNDPs) to dNTPs, is tightly regulated. The homodimer Rnr1p and the heterodimers Rnr2p and Rnr4p, which compose the Rnr complex, are transcriptionally repressed by Crt1 [2,3] while Rnr1p contains binding sites for dATP allosteric inhibition [4]. In the absence of DNA damage, negative regulators such as Sml1p and Dif1p regulate Rnr complex activity through inhibition of the Rnr subunit, Rnr1p, and by subcellular compartmentalization of the Rnr2p-Rnr4p subunits to the nucleus [5,6]. However, in response to DNA damage or replication arrest, Sml1p and Dif1p are phosphorylated and degraded [5,7]. This, along with the transcriptional induction of RNRs and localization of the Rnr complex to the cytoplasm, serves to increase dNTP pools $[5,7,8]$. Checkpoint mediated 
response to DNA damage and replication arrest has been studied extensively, however we do not fully understand how cells recover from checkpoint arrest and downregulate Rnr activity to maintain genome stability.

In this paper, we examined the role of Hug1p (Hydroxyurea, Ultraviolet, Gamma) as a negative regulator of the MEC1 pathway. HUG1 is one of the most differentially expressed genes identified in a screen for gene expression in response to HU treatment [9]. Unlike SML1 and DIF1, the transcription of HUG1 is induced in cells treated with $\mathrm{HU}$ or exposed to ultraviolet or gamma radiation in a MEC1-dependent manner [2]. A deletion of HUG1 has been shown to rescue lethality due to a MEC1 null allele and suppress the HU sensitivity of dun1 $\Delta$ mutants [2]. Studies with HUG1 have primarily focused on its transcriptional response to replication arrest and DNA damage [2,10-13]. Using a polyclonal serum to Hug1p, we have shown that HUG1 encodes for a protein. Our results for HUG1 overexpression phenotypes, a delayed induction pattern of Hug1p in response to HU treatment, and the MEC1-dependent compartmentalization of Hug1p in response to replication arrest define a novel role for Hug1p as a negative regulator of the MEC1-checkpoint response through its compartmentalization with Rnr2p-Rnr4p [14].

\section{Materials and methods}

2.1. Strains, plasmids, cell cycle arrest with $H U$ and growth sensitivity to $H U, M M S$ and BLM

Strains and plasmids are described in Supplementary Table 1. Transformations, cloning, and cell culture were performed using standard methods as previously described [2,15-17]. Additional strain and expression vector construction procedures are outlined in the supplementary materials and methods. Primer sequences are available upon request. Cell cycle arrest with $0.1 \mathrm{M} \mathrm{HU}$ (Fluka Chemika) was as described [2]. Cells ( $>90 \%$ ) exhibited a large budded phenotype with S-phase DNA content, as determined by flow cytometry using an Accuri C6 flow cytometer (BD Accuri Cytometers) [18]. Serial dilutions of cells grown in medium selective for the plasmid were assayed for growth with dextrose (2\%) or raffinose (2\%) plus galactose (2\%) with $0.1 \mathrm{M} \mathrm{HU}, 0.01 \% \mathrm{MMS}$ (Sigma-Aldrich), or $5 \mathrm{mU} / \mathrm{mL}$ BLM (Bristol-Myers Squibb) as described [18].

\subsection{Western blots and subcellular fractionation}

Western blots for Hug1p, Hug1p-GFP, Rnr3p-HA, Sir2p, Pgk1p, Tub2p and subcellular fractionation were performed as described $[18,19]$ using anti-HA (12CA5 Roche), -GFP (A11122 Invitrogen), Sir2p (yN-19) (sc-6666 Santa Cruz), -Pgk1p (459250 Invitrogen), -Hug1p and -Tub2p (antisera generated in Basrai Laboratory).

\subsection{Localization of Hug1p-GFP}

Hug1p-GFP expressing strains were grown to exponential phase in YPD and treated with $0.1 \mathrm{M} \mathrm{HU}$ for $3.5 \mathrm{~h}$. For localization of GAL1-HUG1-GFP, cells were grown to exponential phase in synthetic medium with raffinose (2\%) followed by growth in galactose (2\%) medium for $2 \mathrm{~h}$, shifted to dextrose (2\%) medium with or without $0.1 \mathrm{M}$ HU for $3.5 \mathrm{~h}$. Harvested cells were prepared for microscopy as described [20] except that paraformaldehyde was the only fixative and Hoechst 33342 (Thermo Scientific) was used for nuclear staining. Images were acquired using a Zeiss Axio Observer Z1 microscope.

Image deconvolution and analysis were performed in ImageJ using plug-ins Diffraction PSF 3D to calculate the point-spreadfunction and Iterative Deconvolve 3D [21] for deconvolution.
Localization analysis was performed as described [22]. Cytoplasmic localization was determined empirically to be a nuclear-to-cytoplasmic intensiometric ratio below 0.9 , even distribution between 0.9 and 1.1, and nuclear localization above 1.1. For each strain, at least 100 large budding cells with a nucleus at the bud neck were counted.

Statistical analysis on the subcellular localization data was performed in SAS 9.3 using three-way factorial analysis of variance (ANOVA) with Tukey's multiple comparison range test. Additional statistical analysis is provided in the supplementary materials and methods.

\section{Results}

\subsection{Overexpression of HUG1 increases the sensitivity of wild-type strains to $H U, M M S$ and $B L M$}

We have previously shown that a deletion of HUG1 suppresses the viability of mec $1 \Delta$ strains and HU sensitivity of dun $1 \Delta$ strains [2]. Similar results have been reported in the HUG1 paralogs, DIF1 and SML1, both of which are negative regulators of the Rnr complex and the checkpoint response $[5,7,16]$. To determine if Hug1p acts as a negative regulator of the MEC1 pathway, wild-type strains overexpressing HUG1 were assayed for growth on media containing HU and DNA damaging agents. GAL1-HUG1 was found to increase the sensitivity of wild-type strains to HU on medium containing galactose (GAL) and 0.15 M HU (Fig. 1A, Row 2). GAL1HUG1 or vector (Fig. 1A, Rows 2 and 1, respectively) did not show growth defects on dextrose (DEX) plates with and without HU and GAL plates without HU. The phenotype was specifically due to expression of Hug1p, as a frame-shift mutation in the HUG1 open reading frame (GAL1-HUG1*) abolished the dosage lethality phenotype (data not shown).

Since the viability of mec $1 \Delta$ strains is suppressed by sml1-1 or sml1 $4[5,7,16]$, the dosage lethality of GAL1-HUG1 in wild-type strains was examined for dependence on SML1.Similar to the wild-type strain, the sml1A strain with GAL1-HUG1 exhibited growth inhibition on HU containing medium (Fig. 1A, Row 6). Results verifying that SML1 is not required for the dosage lethality of strains containing GAL1-HUG1 are supported by recent work describing the ubiquitylation and subsequent degradation of Sml1p in response to DNA damage [7]. As expected, the mec1 1 sml1 $\triangle$ strain was sensitive to growth on HU containing plates with or without GAL1-HUG1 (Fig. 1A, Rows 3 and 4). mec1 $\triangle \mathrm{sml1} \Delta$ strains expressing GAL1-HUG1 also showed a slow growth phenotype even in the absence of HU (Fig. 1A, Row 4, center panel). These results are similar to the negative regulator, DIF1, which displays dosage lethality in mec1 $\Delta$ sml1 $\Delta$ strains [5].

In addition to HU sensitivity, GAL1-HUG1 strains exhibited significant growth inhibition on MMS and BLM containing media (Fig. 1B, Row 3) when compared with empty-vector strains (Fig. 1B, Row 2). As expected, the mec1 $\Delta \operatorname{sml1} \Delta$ strain displayed growth inhibition on plates containing MMS and BLM (Fig. 1B, Row 1). Taken together, the synthetic dosage lethality of GAL1HUG1 strains along with previous data support a role for Hug1p as a negative regulator of the MEC1-mediated checkpoint response to DNA damage and replication arrest.

\subsection{Expression of Hug1p shows delayed induction to $0.1 \mathrm{M} \mathrm{HU}$ compared to Rnr3p, a positive effector of the MEC1 pathway}

Using transcriptome profiling, we previously reported that HUG1 represents one of the most highly differentially expressed genes in the yeast genome [2,9]. Initial genome sequencing efforts annotated all ORFs of at least 100 contiguous codons, hence HUG1 was not 
A

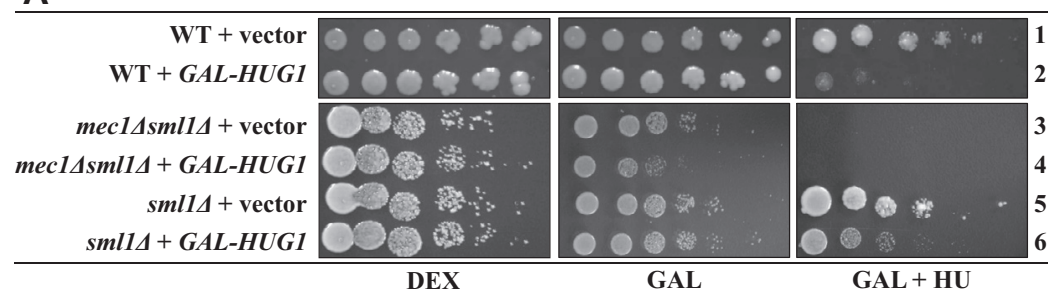

B

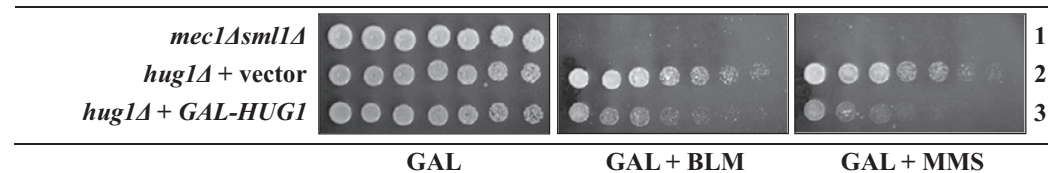

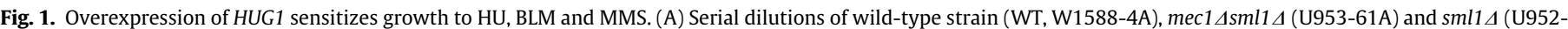

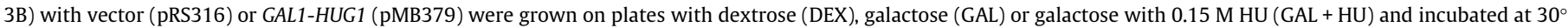

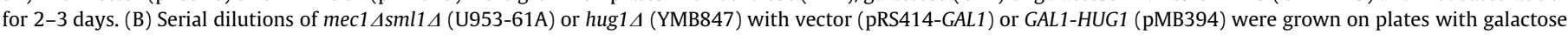
(GAL), galactose with $5 \mathrm{mU} / \mathrm{mL}$ BLM (GAL + BLM), or $0.01 \% \mathrm{MMS}(\mathrm{GAL}+\mathrm{MMS})$ and incubated at $30^{\circ}$ for $2-3$ days.

annotated as it encodes for a protein of 68 amino acids. To validate that HUG1 encodes for a protein, a rabbit polyclonal serum specific to Hug1p was generated. Results from Western blot analysis corroborated results of Northern blot analysis [2], as Hug1p expression was observed in a wild-type strain treated with HU (Fig. 2A, Lane 2). The control includes a hug1 $\Delta$ strain that shows Hug1p expression when transformed with a plasmid expressing HUG1 from its own promoter (pHUG1; Fig. 2A, Lane 6). In agreement with previous results, tup1

A
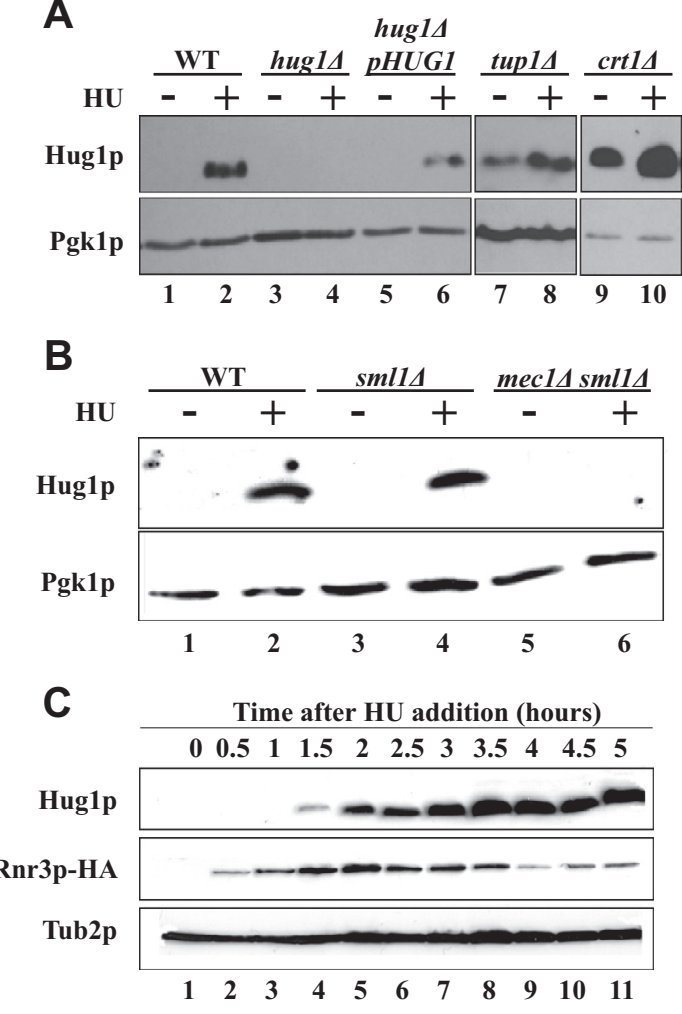

Fig. 2. Genes in the $M E C 1$ pathway are required for HU induced expression of Hug1p and delayed induction of Hug1p compared to Rnr3p. (A) Western blot analysis of wild-type (WT, YPH499), hug1 $\Delta$ (YMB847), hug1 $\Delta$ (YMB847) with pHUG1 (pMB444), tup1 $\Delta$ (Y217), crt1 $\Delta$ (Y577) grown with or without $0.1 \mathrm{M}$ HU for $3.5 \mathrm{~h}$ and probed with anti-Hug1p or -Pgk1p (loading control). (B) Western blot analysis of wild-type (WT, W1588-4A), sml1 $\Delta$ (U952-3C), mec1 $\Delta$ sml1 $\Delta$ (U953-61D) grown with or without $0.1 \mathrm{M}$ HU for $3.5 \mathrm{~h}(\mathrm{C})$ Western blot analysis of RNR3-HA strains (YMB1657) after treatment with $0.1 \mathrm{M}$ HU for various times and probed with anti-Hug1p, -HA (Rnr3p-HA) and -Tub2p (loading control). and $\operatorname{crt1} \Delta$ strains constitutively expressed Hug1p (Fig. 2A, Lanes 7-10). Crt1p, Tup1p and Ssn6p are transcriptional repressors that bind to X-box sequences in the promoter of HUG1 and RNRs in the absence of DNA damage and replication arrest [2]. The polyclonal serum also showed that, in agreement with previous Northern blot analysis, no $\mathrm{HU}$ induced expression of Hug1p was detected in mec1 $\Delta$ sml1 $\Delta$ strains (Fig. 2B, Lane 6) and SML1 was not required for the expression of Hug1p (Fig. 2B, Lane 4).

To gain further insight into the role of Hug1p, HU induced expression of Hug $1 \mathrm{p}$ was compared with Rnr3p, a positive regulator of the MEC1 pathway. Hug1p expression was detected $1.5 \mathrm{~h}$ post-HU addition and increased until approximately $3.5 \mathrm{~h}$ post-HU addition after which no further induction was apparent (Fig. 2C). Consistent with previous reports [3], Rnr3p-HA was detected 30 min post-HU addition, increased until 90-120 min post-HU addition, and subsequently declined (Fig. 2C). The delayed induction of Hug1p with high levels present at 3.5-5 h post-HU addition resembles the profile of Crt1p [3], a negative regulator of RNRs and HUG1 gene expression.

\subsection{Hug1p-GFP localizes to the cytoplasm in HU treated cells}

The subcellular localization of Hug1p-GFP was analyzed by fusing GFP to the C-terminus of Hug1p expressed from its native promoter at the chromosomal locus in the genome. Western blot analysis showed expression of Hug1p-GFP in cells treated with HU (Fig. 3A). Fluorescence microscopy of Hug1p-GFP cells without HU treatment showed only background fluorescence (Fig. 3B, left column). However, upon treatment with HU, Hug1p-GFP was enriched in the cytoplasm and was notably excluded from the nucleus in $96.3 \pm 3.1 \%$ of the cells (Fig. 3B, right column). DNA content measurement by FACS and nuclear morphology of the cells confirmed S-phase arrest of the HU treated cells (data not shown).

To rule out artifacts in localization due to GFP tagging of Hug1p, the data were corroborated by subcellular fractionation of cells expressing non-epitope tagged Hug1p expressed from its native promoter. Total, nuclear (Nuc) and cytoplasmic (Cyto) fractions of cells with or without HU were analyzed by Western blot using anti-Sir2p (nuclear marker), -Pgk1p (cytoplasmic marker) or Hug1p. Sir2p was enriched in the nuclear fraction (Fig. 3C, Lane 5) and Pgk1p in the cytoplasmic fraction (Fig. 3C, Lane 6) in HU treated cells. Hug1p was only observed in the cytoplasmic fraction of the HU treated cells and was excluded from the nucleus (Fig. 3C, Lane 6). The enrichment of Hug1p in the cytoplasmic fraction supports the data showing Hug1p localization to the cytoplasm in $\mathrm{HU}$ treated cells. 
A

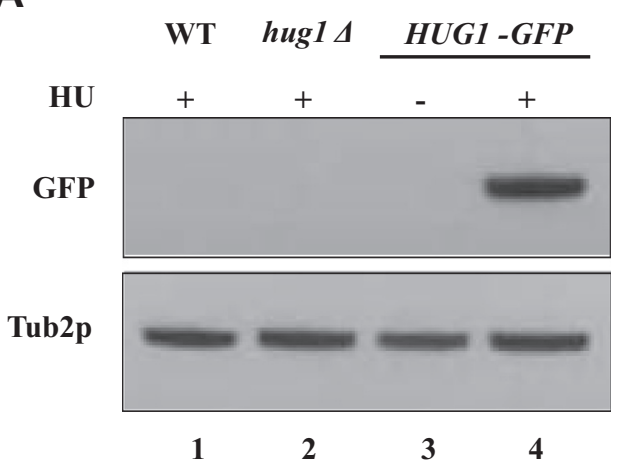

B

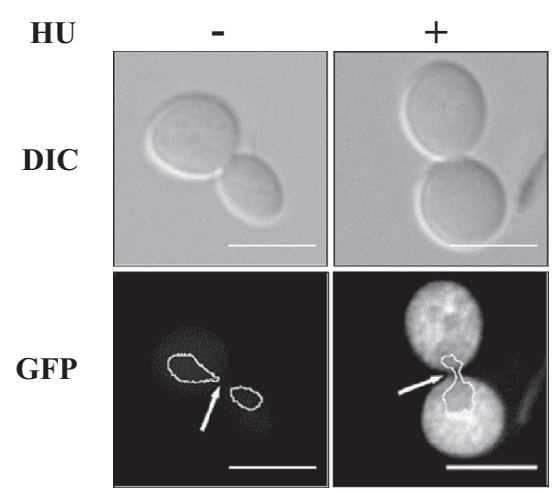

C

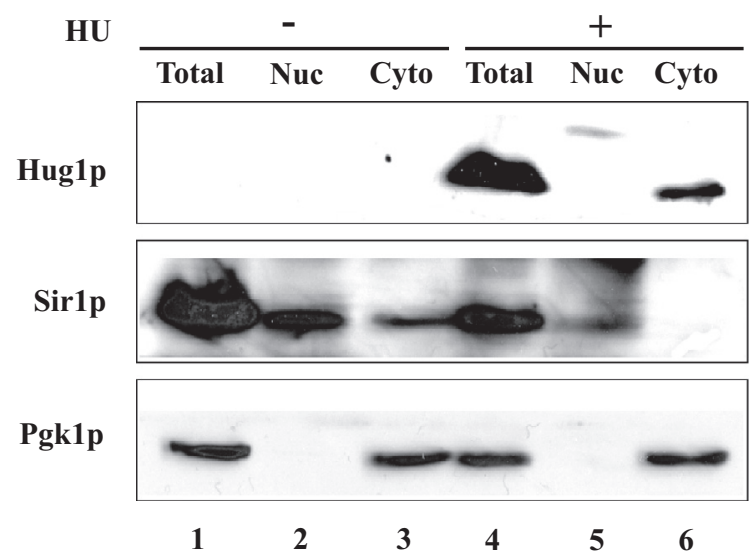

Fig. 3. Hug1 p-GFP is localized to the cytoplasm in response to HU treatment. (A) Western blot analysis showing HU induced expression of HUG1-GFP expressed from its native promoter at the endogenous locus in wild-type (WT, YPH499), hug1 $\Delta$ (YMB847), and HUG1-GFP (YMB1618) strains grown with or without $0.1 \mathrm{M} \mathrm{HU}$ for $3.5 \mathrm{~h}$ and probed with anti-GFP or Tub2p (loading control). (B) Cells expressing Hug1p-GFP grown in the absence (left column) or presence of $0.1 \mathrm{M} \mathrm{HU}$ for $3.5 \mathrm{~h}$ (right column) show exclusion from the nucleus. Arrow indicates bud neck; white line outlines the nucleus; white scale bar length is $5 \mu \mathrm{m}$. (C) Total, nuclear (Nuc) and cytoplasmic (Cyto) fractions of wild-type strains (YPH499) grown in the absence or presence of $0.1 \mathrm{M} \mathrm{HU}$ for $3.5 \mathrm{~h}$ analyzed by Western blot using a polyclonal serum to Hug1p, Sir2p (Nuc), or Pgk1p (Cyto).

3.4. Cytoplasmic localization of Hug1p in response to HU treatment is not merely due to overexpression of the protein

Since HUG1 expression is induced in response to DNA damage and replication arrest in a checkpoint dependent manner [2], we examined if the cytoplasmic localization of Hug $1 \mathrm{p}$ under these conditions may reflect its high level of expression using cells expressing GAL1-HUG1-GFP. Western blot analysis showed that GAL1-HUG1-GFP was expressed in wild-type, mec1 $\Delta$ sml1 $\Delta$, and sml1 $\Delta$ strains grown in galactose medium (Fig. 4A). Cells grown in the presence of galactose for $2 \mathrm{~h}$, followed by growth in glucose medium with or without HU were examined for nuclear morphology and localization of GAL1-HUG1-GFP. Nuclear-to-cytoplasmic intensiometric ratios were quantified as described [22] to determine Hug1p-GFP subcellular compartmentalization for all strains (Fig. S1). In the absence of HU, Hug1p-GFP was primarily localized to the nucleus $(88.2 \pm 2.0 \%)$ whereas a majority of the HU treated cells $(86.6 \pm 3.3 \%)$ exhibited cytoplasmic localization (Fig. 4B, WT). The similar localization pattern of GAL1-HUG1-GFP to that of HUG1-GFP expressed under the native HUG1 promoter revealed that the cytoplasmic localization of Hug1p-GFP is independent of protein expression levels.

\subsection{Cytoplasmic localization of Hug1p-GFP is MEC1-dependent and coincides with the compartmentalization of Rnr2p-Rnr4p to the cytoplasm}

Since genes in the MEC1 pathway are required for the DNA damage and replication arrest induced expression of Hug1p [2], we examined whether the cytoplasmic localization of Hug1p is dependent on the MEC1 effector kinases, MEC1, RAD53, and DUN1. In the absence of $\mathrm{HU}$, Hug1p-GFP mainly localized to the nucleus in mec1 $\Delta$ sml1 $\Delta$ strains $(92.1 \pm 8.5 \%)$, similar to that observed in wild-type strain $(88.2 \pm 2.0 \%)$. However, contrary to the cytoplasmic localization of Hug1-GFP in wild-type cells ( $86.6 \pm 3.3 \%$ ), very few of the mec1 $\Delta$ sml1 $\Delta$ cells showed localization to the cytoplasm $(8.5 \pm 1.1 \%)$ in response to HU treatment. The majority of Hug $1 \mathrm{p}-$ GFP in the mec $1 \Delta$ sml1 $\Delta$ cells was nuclear $(64.0 \pm 7.3 \%)$ or was evenly distributed throughout the cell $(27.5 \pm 7.0 \%)$. The localization pattern observed in mec $1 \Delta \operatorname{sml} 1 \Delta$ strains was independent of SML1 as sml1 $\Delta$ strains exhibited a localization pattern more closely resembling wild-type strains. In the sml1 $\Delta$ strain, Hug1p-GFP localized to the nucleus $(97.1+1.0 \%)$ in the absence of $\mathrm{HU}$ and to the cytoplasm $(85.5 \pm 2.3 \%)$ in the presence HU (Fig. 4B).

We next examined the localization of GAL1-HUG1-GFP in rad53 $\triangle$ and dun $1 \Delta$ strains. In the absence of $\mathrm{HU}$, both rad53 $\Delta$ $(74.8 \pm 11.2 \%)$ and dun1 $\Delta(83.9 \pm 0.5 \%)$ strains showed nuclear localization of Hug1p-GFP similar to that observed in the wild-type strain (Fig. 4B). However, unlike the wild-type cells, in the presence of $\mathrm{HU}$, only a small fraction of rad53 $\Delta$ cells localized to the cytoplasm $(15.7 \pm 4.9 \%)$ with a majority of the cells exhibiting an even distribution $(46.3 \pm 4.5 \%)$ or nuclear localization $(37.9 \pm 9.3 \%)$ of Hug1p-GFP signal. In the presence of $\mathrm{HU}$, the dun1A strains exhibited a cytoplasmic localization profile of Hug1p-GFP that was intermediate to the pattern in mec1 $\Delta$ sml1 $\Delta$ and wild-type strains. Cytoplasmic localization was observed in approximately half the population $(52.5 \pm 5.0 \%)$ of dun $1 \Delta$ cells whereas the remaining cells had either an even distribution $(45.3 \pm 4.1 \%)$ or nuclear localization $(1.9 \pm 0.9 \%)$ of Hug1p-GFP. Taken together, these data indicate that Hug1p-GFP localizes to the cytoplasm in response to HU treatment and this localization is dependent on MEC1, RAD53, and DUN1 and is independent of SML1.

\section{Discussion}

Checkpoint mediated recovery from DNA damage and replication arrest is in part mediated by stringent regulation of Rnr activity. Negative effectors of the MEC1 pathway, namely SML1 and DIF1, interact with Rnr complex subunits and regulate its activity and subcellular compartmentalization. The downregulation of Dif1p and Sml1p in response to DNA damage or replication arrest increases dNTP pools [5-7]. However, after recovery from check- 
A

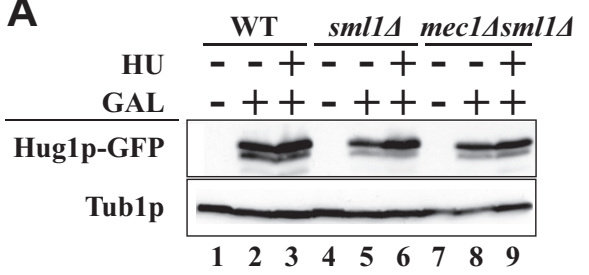

B

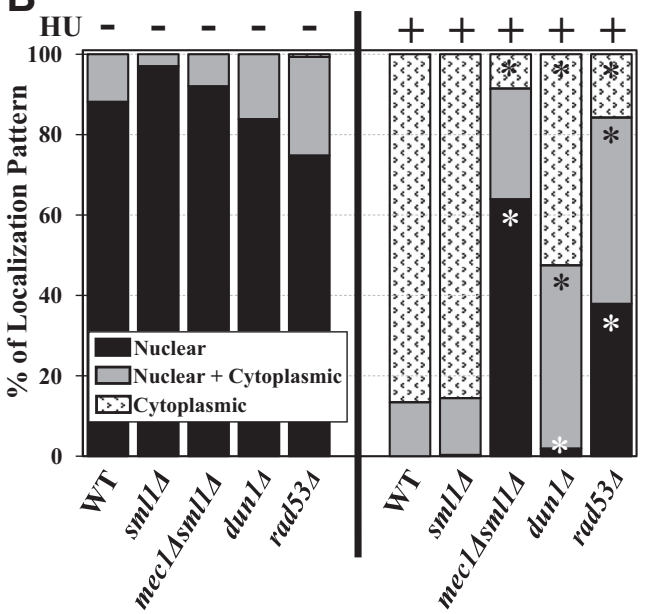

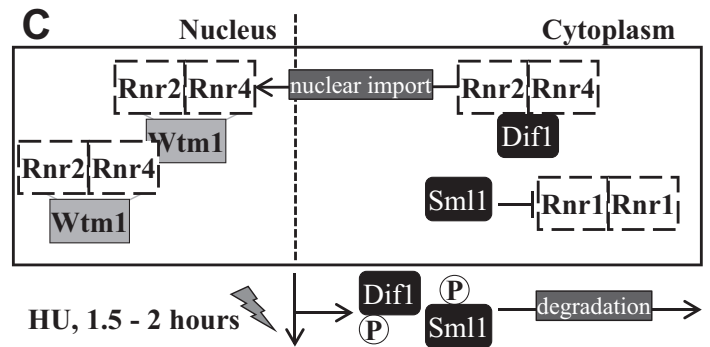

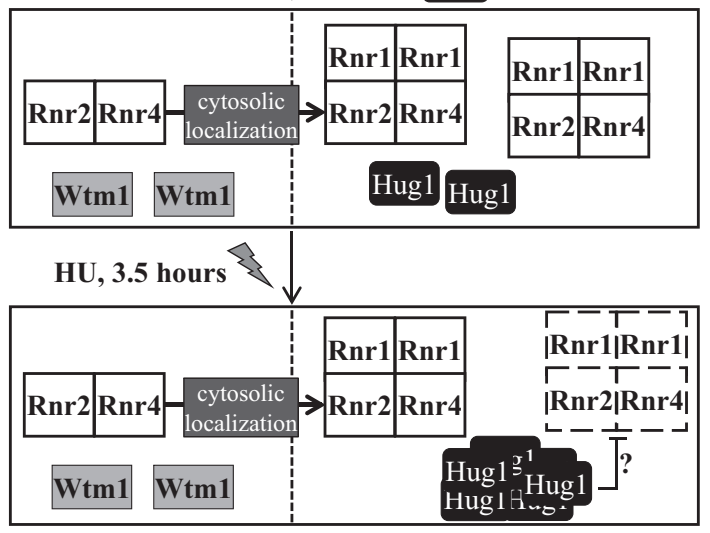

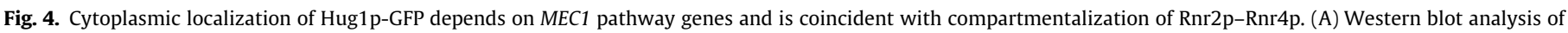

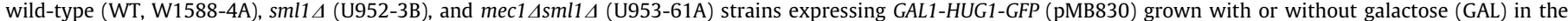

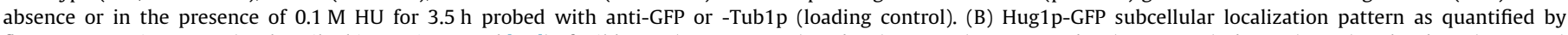

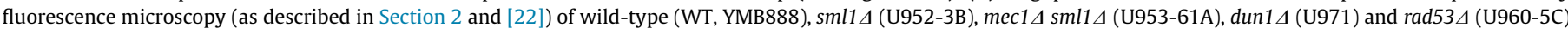

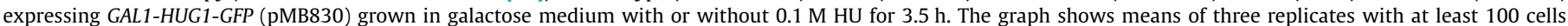

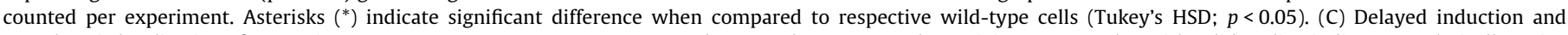

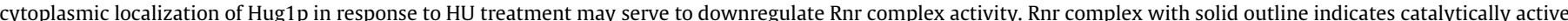

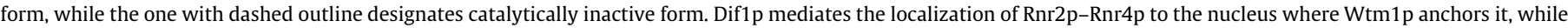

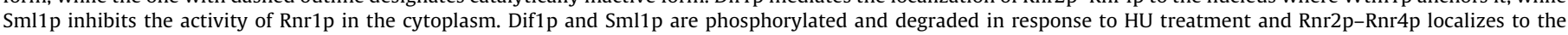

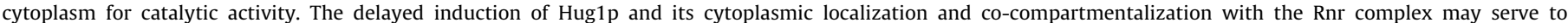
downregulate Rnr activity and facilitate recovery from checkpoint response.

point arrest, Rnr activity must be attenuated by negative regulators for normal cell cycle progression [5-8,16,23-25]. We propose that Hug1p is a negative regulator of the MEC1 pathway, which unlike DIF1 and SML1, is induced in response to DNA damage and replication arrest. This is based on our results which show that: (a) strains expressing GAL1-HUG1 are sensitized to growth in the presence of HU and DNA damaging agents, (b) the temporal pattern of Hug $1 \mathrm{p}$ expression in the presence of HU exhibits a lag when compared with Rnr3p, a positive regulator of the MEC1 pathway, and resembles that of Crt1p, a negative regulator of the MEC1 pathway, and (c) suppression of lethality of mec1 $\Delta$ and HU sensitivity of dun1 $\Delta$ strains by deletion of HUG1.

We propose that Hug1p may serve to negatively regulate the MEC1 pathway by co-compartmentalization with Rnr2p-Rnr4p to the cytoplasm in response to HU treatment. The cytosolic localization is not simply due to overexpression of Hug1p as corroborated by localization analysis of GAL1-HUG1-GFP. Consistent with a requirement of MEC1 pathway genes for the induction of HUG1, cytoplasmic localization of Hug1p was dependent on MEC1, RAD53 and DUN1. Interestingly, Hug1p and Rnr2p-Rnr4p subcellular compartmentalization data share similar dependencies on the MEC1 pathway genes [14]. The localization to the same cellular compartment may allow Hug1p to interact with Rnr2p-Rnr4p through an undetermined, potentially inhibitory mechanism (Fig. 4C) and downregulate Rnr activity. As seen in the model, in cycling cells, Dif1p mediates the localization of Rnr2p-Rnr4p to the nucleus where Wtm1p anchors it, while Sml1p inhibits the activity of Rnr1p in the cytoplasm. After 1.5-2 h of HU induction, Dif1p and Sml1p are phosphorylated and degraded; Rnr2p-Rnr4p is exported from the nucleus to the cytoplasm where it forms the active Rnr complex with the Rnr1p homodimer. After $3.5 \mathrm{~h}$ of $\mathrm{HU}$ treatment, the high level of Hug1p expression and its localization to the cytoplasm and co-compartmentalization with the Rnr complex serve to downregulate Rnr activity and, potentially, dNTP pools. The delayed expression of Hug1p to replication arrest and co-compartmentalization with Rnr2p-Rnr4p may act to negatively regulate Rnr activity in the absence of negative $M E C 1$ effectors, DIF1 and SML1, and permit cellular recovery in post-stress conditions. Taken together, our data define a novel role for HUG1 in the DNA damage and replication arrest pathway.

\section{Acknowledgments}

The authors gratefully acknowledge Sushma Sharma and Andrei Chabes for helpful discussions. W.B.A. was supported by a Donald Clayton Assistantship. M.G.B. was supported by a Ralph E. Powe Enhancement Award and a FIER grant from the Longwell Family Foundation. Support for M.A.B. was provided by the Intramural Research Program of the National Cancer Institute, National Institutes of Health.

\section{Appendix A. Supplementary data}

Supplementary data associated with this article can be found, in the online version, at http://dx.doi.org/10.1016/j.bbrc.2013.08.089. 


\section{References}

[1] K.A. Nyberg, R.J. Michelson, C.W. Putnam, T.A. Weinert, Toward maintaining the genome: DNA damage and replication checkpoints, Annu. Rev. Genet. 36 (2002) 617-656.

[2] M.A. Basrai, V.E. Velculescu, K.W. Kinzler, P. Hieter, NORF5/HUG1 is a component of the MEC1-mediated checkpoint response to DNA damage and replication arrest in Saccharomyces cerevisiae, Mol. Cell. Biol. 19 (1999) 7041 7049.

[3] M. Huang, Z. Zhou, S.J. Elledge, The DNA replication and damage checkpoint pathways induce transcription by inhibition of the Crt1 repressor, Cell 94 (1998) 595-605.

[4] H.-H.T. Nguyen, J. Ge, D.L. Perlstein, J. Stubbe, Purification of ribonucleotide reductase subunits Y1, Y2, Y3, and Y4 from yeast: Y4 plays a key role in diiron cluster assembly, PNAS 96 (1999) 12339-12344.

[5] Y.D. Lee, J. Wang, J. Stubbe, S.J. Elledge, Dif1 is a DNA-damage-regulated facilitator of nuclear import for ribonucleotide reductase, Mol. Cell 32 (2008) 70-80.

[6] X. Wu, M. Huang, Dif1 controls subcellular localization of ribonucleotide reductase by mediating nuclear import of the R2 subunit, Mol. Cell. Biol. 28 (2008) 7156-7167.

[7] B.L. Andreson, A. Gupta, B.P. Georgieva, R. Rothstein, The ribonucleotide reductase inhibitor, Sml1, is sequentially phosphorylated, ubiquitylated and degraded in response to DNA damage, Nucleic Acids Res. 38 (2010) 64906501.

[8] X. Zhao, R. Rothstein, The Dun1 checkpoint kinase phosphorylates and regulates the ribonucleotide reductase inhibitor Sml1, PNAS 99 (2002) 37463751.

[9] V.E. Velculescu, L. Zhang, W. Zhou, J. Vogelstein, M.A. Basrai, D.E. Bassett Jr., P. Hieter, B. Vogelstein, K.W. Kinzler, Characterization of the yeast transcriptome, Cell 88 (1997) 243-251.

[10] T. Wei, C. Zhang, X. Xu, M. Hanna, X. Zhang, Y. Wang, H. Dai, W. Xiao, Construction and evaluation of two biosensors based on yeast transcriptional response to genotoxic chemicals, Biosens. Bioelectron. 44 (2013) 138-145.

[11] W.B. Ainsworth, C.M. Rome, M.A. Hjortso, M.G. Benton, Construction of a cytosolic firefly luciferase reporter cassette for use in PCR-mediated gene deletion and fusion in Saccharomyces cerevisiae, Yeast 29 (2012) 505-517.

[12] M.G. Benton, N.R. Glasser, S.P. Palecek, The utilization of a Saccharomyces cerevisiae HUG1P-GFP promoter-reporter construct for the selective detection of DNA damage, Mutat. Res. 633 (2007) 21-34.
[13] V.M. Sharma, R.S. Tomar, A.E. Dempsey, J.C. Reese, Histone deacetylases RPD3 and HOS2 regulate the transcriptional activation of DNA damage-inducible genes, Mol. Cell. Biol. 27 (2007) 3199-3210.

[14] R. Yao, Z. Zhang, X. An, B. Bucci, D.L. Perlstein, J. Stubbe, M. Huang, Subcellular localization of yeast ribonucleotide reductase regulated by the DNA replication and damage checkpoint pathways, PNAS 100 (2003) 6628-6633.

[15] A. Adams, D.E. Gottschling, C.A. Kaiser, T. Stearns, Methods in Yeast Genetics Cold Spring Harbor Laboratory, Cold Spring Harbor, New York, 1997.

[16] X. Zhao, E.G.D. Muller, R. Rothstein, A suppressor of two essential checkpoint genes identifies a novel protein that negatively affects dNTP pools, Mol. Cell 2 (1998) 329-340.

[17] D. Mumberg, R. Muller, M. Funk, Regulatable promoters of Saccharomyces cerevisiae: comparison of transcriptional activity and their use for heterologous expression, Nucleic Acids Res. 22 (1994) 5767-5768.

[18] C.D. Carter, L.E. Kitchen, W.-C. Au, C.M. Babic, M.A. Basrai, Loss of SOD1 and LYS7 sensitizes Saccharomyces cerevisiae to hydroxyurea and DNA damage agents and downregulates MEC1 pathway effectors, Mol. Cell. Biol. 25 (2005) 10273-10285.

[19] C. Liang, B. Stillman, Persistent initiation of DNA replication and chromatinbound MCM proteins during the cell cycle in cdc6 mutants, Genes Dev. 11 (1997) 3375-3386.

[20] J.R. Pringle, A.E. Adams, D.G. Drubin, B.K. Haarer, Immunofluorescence methods for yeast, Methods Enzymol. 194 (1991) 565-602.

[21] R.P. Dougherty, Extensions of DAMAS and benefits and limitations of deconvolution in beamforming, in: Proc. 11th AIAACEAS Aeroacoustics Conference, AIAA-2005, 2005.

[22] L. Malinovska, S. Kroschwald, M.C. Munder, D. Richter, S. Alberti, Molecular chaperones and stress-inducible protein-sorting factors coordinate the spatiotemporal distribution of protein aggregates, Mol. Biol. Cell 23 (2012) 3041-3056.

[23] A. Chabes, B. Stillman, Constitutively high dNTP concentration inhibits cell cycle progression and the DNA damage checkpoint in yeast Saccharomyces cerevisiae, PNAS 104 (2007) 1183-1188.

[24] E. Kim, W. Siede, Phenotypes associated with Saccharomyces cerevisiae Hug protein, a putative negative regulator of dNTP Levels, reveal similarities and differences with sequence-related Dif1, J. Microbiol. 49 (2011) 78-85.

[25] J. Poli, O. Tsaponina, L. Crabbe, A. Keszthelyi, V. Pantesco, A. Chabes, A. Lengronne, P. Pasero, DNTP pools determine fork progression and origin usage under replication stress, EMBO J. 31 (2012) 883-894. 